\title{
Feasibility Study of INS for Upper Wind Measurement
}

\author{
Tao Ye, Zhao Shijun \\ Institute of Meteorology and Oceanology \\ PLA Univ. of Sci. and Tech. \\ Jiangsu, Nanjing 211101 \\ taoye1992@163.com
}

\begin{abstract}
Upper wind data is the basic data for meteorological services and military supporting missions, and balloon-tracing method is the most commonly used method in meteorological operation. In this paper a balloon-tracing and wind measuring method bases on inertial navigation systems (INS) is proposed accounting to the limitation in conventional methods. In this paper, INS measurement for upper wind system is established, its mathematical model is built, and the errors are analyzed. The results indicated that the average relative errors of wind velocity ranges from $2 \%$ to $4 \%$ and average wind direction measurement ranges from 7 degrees to 14 degrees; The maximum relative errors of wind velocity measurement ranges from $8 \%$ to $11 \%$, the maximum errors of wind direction measurement ranges from 12 degrees to 17 degrees. Above all, the application of INS in upper wind measurement is feasible.)
\end{abstract}

\section{Index Terms - SINS, Upper Wind Measurement, MEMS.}

\section{INTRODUCTION}

Upper Wind measurement generally is the measurement of horizontal air flow, including the wind velocity and direction ranging from ground to the altitude of more than $30 \mathrm{~km}$. Realtime and accurate upper wind data is the fundamental data of weather forecasts and the supporting of bombardment, air drop, aircraft landing and taking-off, rocket or missile launching and artillery ballistic correction. Balloon-tracing method is the most commonly used method in meteorological operation.

Currently, the upper wind is usually measured by following equipment ${ }^{[1]}$ : optical theodolites, radio theodolites, radars and GNSS (Global Navigation Satellite System). Measurement based on optical theodolites is limited to low visibility or lowcloud so it can't assure the measurement altitude; Radio theodolites and radars are big and heavy, also, the measurement errors increase with the altitude; The Radars emit high power electromagnetic wave which leads to poor concealment of electromagnetism and low battlefield survivability; GNSS based navigation measurements have high accuracy, small size and low weight, but GNSS wind measurements highly rely on navigation satellites and are easy to jam, which leads to insecurity in battle field environment.

Inertial navigation technology solves the position and velocity of a moving object by integrating the inertial (acceleration) information of itself. An Inertial navigation system (INS) is hard to be traced or jammed because it neither relies on outside information nor emits high power electromagnetic wave when it's working, which has been widely used in precision guide weapons and Unmanned Aerial Vehicles (UAV). Currently, INS's cost is reducing and performance is increasing. With its advantages of independence, noninterference, precision and concealment of electromagnetism, this paper proposed a method of upper wind measurement bases on INS technology and its operating procedure. This paper also studied in the cost, size, weight and working environment of the INS to figure out the feasibility of an INS based upper wind measurement method.

\section{THE MOdEL OF INS FOR WIND MEASUREMENT SYSTEM}

An INS solves the vehicle's acceleration relative to the ground $\mathbf{a}_{t}(t)$ according to the acceleration signal acquired by accelerometers and navigation attitude signal acquired by electronic compasses or gyroscopes. Vehicle's position can be solved by dual integral $\mathbf{a}_{t}(t)$ :

$$
\mathbf{X}(t)=\int_{0}^{t} \int_{0}^{t} \mathbf{a}_{t}(t) \mathrm{dt}^{2}
$$

An INS usually consists of Inertial Measurement Unit (IMU) which consists of accelerometers which measure translational motions in 3 axes and gyroscopes or electronic compasses which measure rotational motions around 3 axes, computing unit which solves speed and position of the vehicle according to the acceleration and navigation attitude signal, control and output unit which outputs the measurement results.

\section{A. Install the INS and Choose Vehicle Coordinate System}

INS can be divided into platform INS (PINS) and strapdown INS (SINS), it depends on methods of installation. The former method installs IMU on a platform which maintains a fixed relative attitude to geographic coordinates, the latter method installs IMU on the vehicle directly and moves with it all the time, which is smaller and easier to install but takes up more computing recourses ${ }^{[2]}$. This paper chooses SINS to implement the upper wind measurement and build IMU with accelerometers, gyroscopes and electronic compasses. With IMU installed directly on the radiosonde and their sensitive axes align to the measured vehicle's ( radiosonde's) coordinate system, SINS has greater advantages 
in low weight and small size over PINS. In the measuring process, gyroscopes and electronic compasses measure the sonde's angular acceleration relative to geographic coordinate and come to the coordinate transformation matrix (navigational matrix), with it the computing unit can transforms the acceleration data in sonde's coordinate into geographic coordinate and navigation parameters can be reached according to the acceleration data above. The SINS diagram is shown in figure 1 .

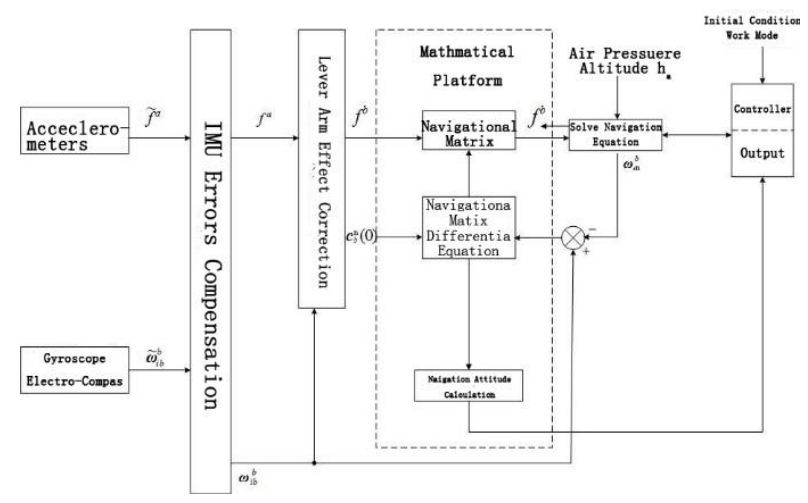

Fig. 1. SINS Based Wind Measurement System Diagram

In Figure 1:

$\tilde{f}^{a}$ is specific force vector acquired by accelerometers;

$\tilde{\omega}_{i b}^{a}$ is angular speed vector acquired by gyroscope and electronic compass;

$f^{a}$ is error compensated specific force vector;

$\omega_{i b}^{a}$ is error compensated angular speed vector ;

Lever arm effect correction compensate the alignment errors of IMUs' axis, and it's done with ground based equipment before sounding process.

First, establish the vehicle coordinate system (b system)

In b system:

The $\mathrm{Z}$ axis of INS point to the zenith;

Uses the Euler angles: $\Psi, \theta, \gamma$;

The yaw angle $\Psi$ is in the direction of $\mathrm{Z}$ axis, its direction follows right-hand rule;

The pitch angle $\theta$ is in the direction of $\mathrm{X}$ axis, its direction follows right-hand rule;

The rolling angle $\gamma$ is in the direction of $\mathrm{Y}$ axis, its direction follows right-hand rule;

The core of SINS is to transform specific forces in vehicle's coordinate $\mathbf{f}_{b}$ into specific forces in geographic coordinate $\mathbf{f}_{\mathbf{t}}$ according to the navigation attitude information, and ultimately solve navigation parameters by integrating $\mathbf{f}_{\mathbf{t}}$.

With the angular signal, SINS can come to the navigation attitude matrix, and with navigation attitude matrix, SINS can transform the sonde's acceleration vectors into geographic coordinate system to perform navigational computing, meanwhile sonde's attitude and course data can also be extracted from this navigational matrix. Thus, solving the attitude matrix, coordinate transformation and extraction of course and attitude information, these 3 functions actually replace the navigation platform in PINS and form up a socalled "mathematical platform"[3], solving angular speed in geographic coordinate of SINS has the same function with the rotation of navigation platform. ${ }^{[4]}$

Navigational equation ${ }^{[5]}$ :

$\dot{\mathbf{v}}_{\mathbf{t}}=\mathbf{f}_{\mathbf{t}}-(2 \boldsymbol{\Omega}+\rho) \times \mathbf{v}_{\mathbf{t}}+\mathbf{g}_{v}$

In equation

$\mathbf{v}_{\mathbf{t}}$ is speed vector of the sonde in geographic coordinate; $\mathbf{f}_{t}=\left[\begin{array}{lll}f_{x} & f_{y} & f_{z}\end{array}\right]^{T}$ is the specific force vector acquired by accelerometers after coordinate transformation and centripetal force compensation; $\boldsymbol{\Omega}$ is the angular speed vector of earth rotation; $\mathbf{g}_{\mathrm{v}}=\mathrm{g}_{\mathrm{m}} \mathbf{\Omega} \times(\boldsymbol{\Omega} \times \mathbf{r})$ is gravitational acceleration vector; $\mathbf{r}$ is position vector of the sonde in inertial space; $g_{\mathrm{m}} \quad$ is terrestrial attraction vector; $\boldsymbol{\Omega} \times(\boldsymbol{\Omega} \times \mathbf{r}) \quad$ is $\quad$ centripetal acceleration; For specific force $\mathbf{f}_{\mathbf{t}}$ :

$\left[\begin{array}{lll}\mathbf{f}_{\mathbf{x}}^{\mathbf{b}} & \mathbf{f}_{\mathbf{y}}^{\mathbf{b}} & \mathbf{f}_{\mathbf{z}}^{\mathbf{b}}\end{array}\right]^{\mathrm{T}}=\mathbf{C}_{\mathbf{n}}^{\mathbf{b}}\left[\begin{array}{lll}\mathbf{f}_{\mathbf{x}} & \mathbf{f}_{\mathbf{y}} & \mathbf{f}_{\mathbf{z}}\end{array}\right]^{\mathrm{T}}$

$\left[\begin{array}{lll}\mathrm{f}_{\mathrm{x}}^{\mathrm{b}} & \mathrm{f}_{\mathrm{y}}^{\mathrm{b}} & \mathrm{f}_{\mathrm{z}}^{\mathrm{b}}\end{array}\right]^{\mathrm{T}}$ is the actual specific force acquired by 3 axes accelerometers or specific force in radiosonde's coordinate system. $\mathbf{C}_{\mathbf{n}}^{\mathbf{b}}$ is the attitude transformation matrix, which is a orthogonal matrix, i.e. $\mathbf{C}_{\mathbf{n}}^{\mathrm{b}}=\left(\mathbf{C}_{\mathrm{b}}^{\mathrm{n}}\right)^{\mathrm{T}}$, and:

$$
\mathbf{C}_{\mathbf{n}}^{\mathrm{b}}=\left[\begin{array}{lll}
\mathbf{C}_{\mathrm{n} 1}^{\mathrm{b}} & \mathbf{C}_{\mathrm{n} 2}^{\mathrm{b}} & \mathbf{C}_{\mathrm{n} 3}^{\mathrm{b}}
\end{array}\right]
$$

In equation 4:

$$
\begin{gathered}
\mathbf{C}_{\mathbf{n} 1}^{\mathbf{b}}=\left[\begin{array}{c}
\cos \gamma \cos \Psi-\sin \gamma \sin \Psi \sin \theta \\
\cos \gamma \sin \Psi+\sin \gamma \cos \Psi \sin \theta \\
-\sin \gamma \cos \theta
\end{array}\right] \\
\mathbf{C}_{\mathbf{n} 2}^{\mathbf{b}}=\left[\begin{array}{c}
-\sin \Psi \cos \theta \\
\cos \Psi \cos \theta \\
\sin \theta
\end{array}\right] \\
\mathbf{C}_{\mathbf{n} 3}^{\mathbf{b}}=\left[\begin{array}{c}
\sin \gamma \cos \Psi+\cos \gamma \sin \Psi \sin \theta \\
\sin \gamma \sin \Psi+\cos \gamma \cos \Psi \sin \theta \\
\cos \gamma \cos \theta
\end{array}\right]
\end{gathered}
$$

The sonde's speed and position can be solved according to this mathematical model, and thus, the upper wind information can be solved according to this information and atmospheric measurement theories.

\section{B. Initial Alignment before measurement}

The initial alignment of INS aims to establish a initial relative relationship between radiosonde's coordinate system and geographic coordinate system, and provides initial value for further integral calculations. This paper uses the following low cost initial alignment algorithm ${ }^{[6]}$.

Before measurement process, the output of 3 axes accelerometers is:

$$
\mathbf{f}_{\mathrm{b}}=\left[\begin{array}{lll}
\mathrm{f}_{\mathrm{x}}^{\mathrm{b}} & \mathrm{f}_{\mathrm{y}}^{\mathrm{b}} & \mathrm{f}_{\mathrm{z}}^{\mathrm{b}}
\end{array}\right]^{\mathrm{T}}
$$

The actual specific force in geographic coordinate is:

$$
\mathbf{f}_{\mathrm{t}}=\left[\begin{array}{lll}
0 & 0 & -\mathrm{g}
\end{array}\right]^{\mathrm{T}}
$$


Initial rolling and pitch angle $\gamma_{0}$ and $\theta_{0}$ is:

$$
\gamma_{0}=-\arctan \left(\frac{\mathrm{f}_{\mathrm{x}}^{\mathrm{b}}}{\mathrm{f}_{\mathrm{z}}^{\mathrm{b}}}\right), \theta_{0}=-\arcsin \left(\frac{\mathrm{f}_{\mathrm{y}}^{\mathrm{b}}}{\mathrm{g}}\right)
$$

Initial yaw angle $\Psi_{0}$ can be provided by electronic compass directly. Thus the initial alignment of a SINS based upper wind measurement system is finished.

\section{INS FOR UPPER WIND MEASUREMENT PERFORMANCE ANALYSIS}

\section{A. INS for wind Measurement System Error Analysis}

The error of SINS accumulates with time considerably because it solves navigation parameters by one or two orders integration. Usually, upper air measurement process can last for about 1 hour. So, the analysis of SINS for upper wind measurement errors is especially important.

The performance of an upper wind measurement system is usually assessed by its position error, wind velocity error and wind direction error. For SINS, the error generally comes from IMU errors, installation errors, initial alignment errors, inference errors and nonlinear errors and so on.

Meanwhile, errors can also be divided into constant errors and non-constant error, constant errors can be eliminated by ground calibration. This paper majorly analyses non-constant error.

The error model ${ }^{[7]}$ :

$$
\begin{aligned}
\delta \dot{\mathbf{v}}_{\mathbf{t}}= & \Delta \mathbf{C}_{\mathbf{b}}^{\mathbf{n}} \times \tilde{\mathbf{f}}^{\mathrm{b}}+\mathbf{C}_{\mathbf{b}}^{\mathbf{n}} \times \delta \mathbf{f}^{\mathrm{b}}-(\mathbf{2} \boldsymbol{\Omega}+\boldsymbol{\rho}) \times \delta \mathbf{v}_{\mathbf{t}} \\
& -(\mathbf{2} \delta \boldsymbol{\Omega}+\delta \boldsymbol{\rho}) \times \mathbf{v}_{\mathrm{t}}^{\mathrm{m}}+\delta \mathbf{g}_{\mathrm{v}}
\end{aligned}
$$

In equation $\Delta \mathbf{C}_{\mathbf{b}}^{\mathbf{n}} \quad$ is attitude matrix error; $\tilde{\mathbf{f}}^{\mathrm{b}}$ is the actual specific force acquired by accelerometers, which includes drift error and scaling factor error; $\delta \mathbf{f}^{\mathrm{b}}$ is the specific force error acquired by accelerometers; $(2 \Omega+\boldsymbol{\rho}) \times \delta \mathbf{v}_{\mathbf{t}}$ is the Coriolis effect term in geographic coordinate;

$(\mathbf{2} \delta \boldsymbol{\Omega}+\delta \boldsymbol{\rho})$ is earth rotation parameter error; $\mathbf{v}_{\mathrm{t}}^{\mathrm{m}}$ is the computed value of vehicle's speed vector; $\delta \mathbf{g}$ is the gravitational parameter error;

\section{B. Components Selection and Simulation Result Analysis of} INS For Upper Wind Measurement System

This paper chooses following components to do the simulation due to the low cost of a radiosonde : (1) ADXL345 digital accelerometers; (2) HMC5883L integrated digital compass; (3) L3G4200D 3 axis digital gyroscope;

This IMU's size and weight can meet the demand of upper air measurement and the total cost is at about $200 \mathrm{RMB}$, and currently, a conventional digital radiosonde can cost about 800 RMB, a GPS radiosonde can cost about $1300 \mathrm{RMB}$, so INS based radiosonde has considerable advantages in price.

IMUs parameters is shown in Table 1.
TABLE I. GENERAL PARAMETERS OF INERTIAL MEASUREMENT UNITS

\begin{tabular}{|c|l|l|l|}
\hline & Accelerometers & \multicolumn{1}{c|}{ Compasses } & \multicolumn{1}{|c|}{ Gyroscopes } \\
\hline Measurement range & $\pm 4 \mathrm{~g}$ & \pm 8 gauss & $\pm 500 \mathrm{dps}$ \\
\hline Nonlinearity & $0.50 \%$ & $0.10 \%$ & $0.20 \%$ \\
\hline Resolution & $10 \mathrm{Bits}$ & $12 \mathrm{Bits}$ & $10 \mathrm{Bits}$ \\
\hline Senstivity & $128 \mathrm{LSB} / \mathrm{g}$ & $\pm 2 \% \mathrm{FS}$ & $\begin{array}{l}12.5 \mathrm{mdps} / \mathrm{dig} \\
\text { it }\end{array}$ \\
\hline 0 drift & $\pm 150 \mathrm{mg}$ & N/A & $\pm 15 \mathrm{dps}$ \\
\hline Noise & $0.75 \mathrm{LSB} \mathrm{rms}$ & N/A & $\begin{array}{l}0.03 \\
\text { dps } / \mathrm{sqrt}(\mathrm{Hz})\end{array}$ \\
\hline $\begin{array}{c}\text { Operating } \\
\text { Temperature }\end{array}$ & $-40^{\circ} \mathrm{C}$ to $80^{\circ} \mathrm{C}$ & $-30^{\circ} \mathrm{C}$ to & $-40^{\circ} \mathrm{C}$ to $85^{\circ} \mathrm{C}$ \\
& & $85^{\circ} \mathrm{C}$ & \\
\hline
\end{tabular}

In table 1, LSB means Least Significant Digit; rms means rootmean-square; dps means degree per second. $g$ means gravitational acceleration;

To assess the performance of the measurement system, this paper simulates the measurement in random generated wind fields. In the simulation the measurement lasts for 1 hour and assumes the radiosonde can completely response to the wind field. Substituted parameters in table 1 into equation (8), the numeric solution $\delta \dot{\mathbf{v}}_{\mathbf{t}}$ is the acceleration error, the 1 order and 2 orders time integration can result in speed error $\delta \mathbf{v}_{\mathbf{t}}$ and position error $\delta \mathbf{r}_{\mathbf{t}}$.

Figure 2,3 and 4 show the simulation results when the average wind velocity is $30 \mathrm{~m} / \mathrm{s}$, and wind direction is $90^{\circ}$ (due west, along $\mathrm{X}$ axis).

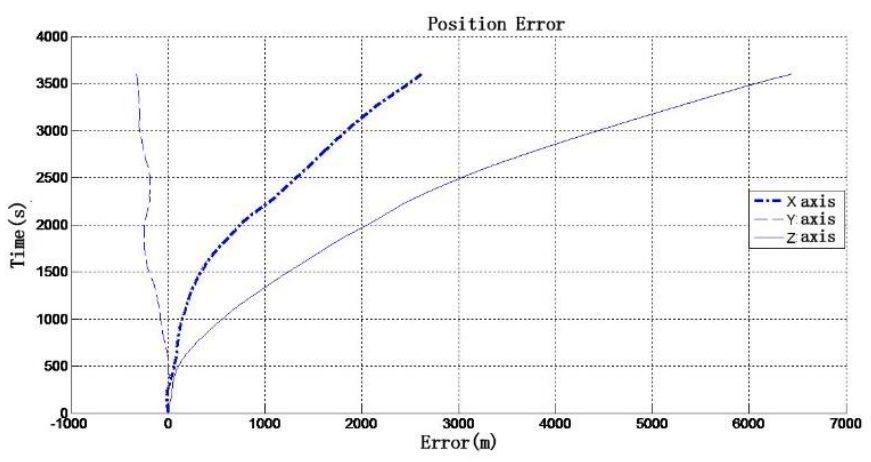

Fig. 2. Positioning Error
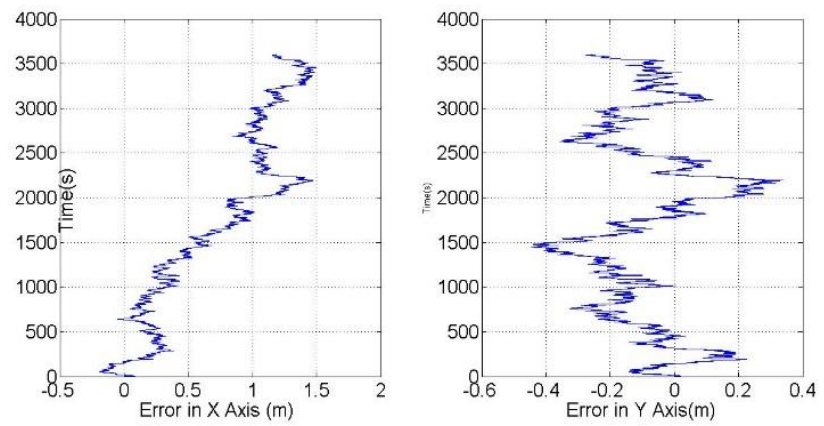

Fig. 3. Wind Velocity Error 

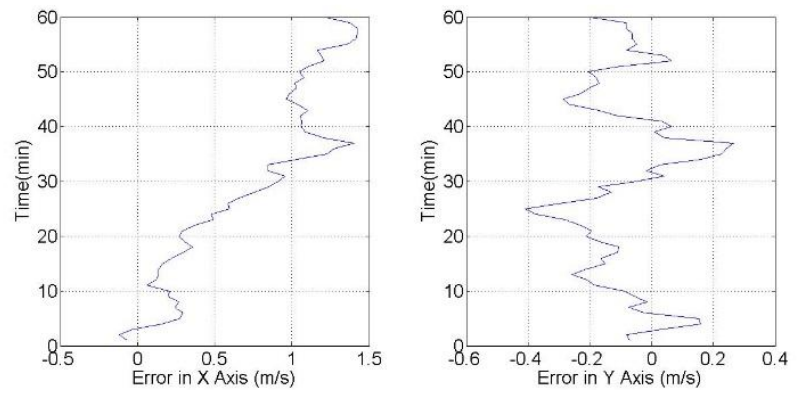

Fig. 4. Layer Wind Velocity Error

Figure 2 shows positioning errors, the measurement system's positioning error accumulates by time considerably, especially the error in $\mathrm{Z}$ axis, so it's hard to trace the system's altitude unless a self-adaptive filter is applied to reduce the error, otherwise altitude data can be solved by air pressure detected by radiosonde.

Figure 3 shows the wind velocity error in 2 horizontal axes. The error in the direction along actual wind direction accumulates slowly and reach $1.5 \mathrm{~m} / \mathrm{s}$ at the end of measurement when the mean velocity of wind is $30 \mathrm{~m} / \mathrm{s}$, the velocity error perpendicular to the actual direction fluctuating around 0 and causes wind direction error. The average direction error is $7^{\circ}$ and maximum direction error is $12^{\circ}$.

Figure 4 shows the layer wind error, which applied the idea of layer wind in atmospheric measurement theory and smoothes the measurement result over time, it can reduce stochastic error at the cost of reducing time resolution. The size of smooth window can be chosen according to actual situations.

Table 2 shows simulation result in variable wind fields.

The simulation results show that the INS of upper wind measurement system's positioning performance is relatively poor, however, in actual upper air measurement operations, the radiosonde's position is not essential when the speed can be solved directly. According to the results shown above, the velocity errors maintained in an acceptable range during the 60 min of simulation. Average relative error of Velocity ranges from $2 \%-4 \%$, average wind direction error ranges from $7^{\circ}-14^{\circ}$, the maximum relative errors of wind velocity measurement ranges from $8 \%-11 \%$ and the maximum errors of wind direction measurement ranges from $12^{\circ}-17^{\circ}$, it can be seen that when the actual wind velocity is high, the accuracy of INS for upper wind measurement can meet the request given in Upper Air Measurement Specification of maximum 10\% velocity error and $10^{\circ}$ direction error ${ }^{[8]}$. But when the velocity is low, the system's accuracy breach the $1 \mathrm{~m} / \mathrm{s}$ velocity error and $10^{\circ}$ direction borderline in Upper Air Measurement Specification, but the average error can still meet the request. If using time smooth to the measurement result, stochastic errors can be further reduced.
TABLE II. SimUlation Result IN VARIABLE Wind Field

\begin{tabular}{|c|c|c|l|}
\hline Mean Velocity & $30 \mathrm{~m} / \mathrm{s}$ & $\mathbf{2 0 m} / \mathrm{s}$ & \multicolumn{1}{|c|}{$\mathbf{1 0 m} / \mathbf{s}$} \\
\hline Wind Direction & $90^{\circ}$ & $180^{\circ}$ & $200^{\circ}$ \\
\hline $\begin{array}{c}\text { Average Velocity } \\
\text { Error }\end{array}$ & $0.733 \mathrm{~m} / \mathrm{s}$ & $0.729 \mathrm{~m} / \mathrm{s}$ & $0.473 \mathrm{~m} / \mathrm{s}$ \\
\hline $\begin{array}{c}\text { Average Direction } \\
\text { Error }\end{array}$ & $7^{\circ}$ & $14^{\circ}$ & $13^{\circ}$ \\
\hline $\begin{array}{c}\text { Average Relative } \\
\text { Velocity Error }\end{array}$ & $2.40 \%$ & $3.60 \%$ & $4.70 \%$ \\
\hline $\begin{array}{c}\text { Maximum Velocity } \\
\text { Error }\end{array}$ & $2.455 \mathrm{~m} / \mathrm{s}$ & $1.849 \mathrm{~m} / \mathrm{s}$ & $1.124 \mathrm{~m} / \mathrm{s}$ \\
\hline $\begin{array}{c}\text { Maximum Direction } \\
\text { Error }\end{array}$ & $12^{\circ}$ & $17^{\circ}$ & $16^{\circ}$ \\
\hline $\begin{array}{c}\text { Maximum Relative } \\
\text { Velocity Error }\end{array}$ & $8.20 \%$ & $9.20 \%$ & $11.20 \%$ \\
\hline
\end{tabular}

\section{CONCLUSION}

This paper applies INS technology into upper wind measurement field for its merits of all-weather-availability, system independence and high accuracy. INS for upper wind measurement system is feasible and it meets demand of the modern battle field environment better than conventional RADAR wind measurement method.

But this measurement system cannot measure the altitude of a radiosonde without self-adaptive filters due to its own disability. The altitude need to be solved by air pressure detected by radiosonde until self-adaptive filter is applied in the system. All in all, account for its size, weight, cost and performance, INS for upper wind measurement system is applicable as an backup method in modern upper wind measurement and is possible to expand the upper air measurement operation's competence.

\section{REFERENCES}

[1] Sun Xuejin, Wang Xiaolei, Li Hao et al, Atomspheric Measurement, 1rd ed. Beijing: Metorological press, 2009, pp 265-299.

[2] Axford.D. N., "On the Accuracy of Wind Measurements Using an Inertial Platform in an Aircraft, and an Example of a Measurement of the Vertical Mesostructure of the Atmosphere", in Journal of Applied Meteorology. vol. 7, Issue 4, pp.645-666.

[3] Li yue, Navigation and positioning, 1rd ed. Beijing: National Defence Industry press, 2008, pp.420-421.

[4] Li Zhen," Research on Data Processing Technique of Inertial Navigation and Orientation System ", Shenyang: Shenyang University of Technology, Graduate's Thesis, 2007.

[5] Li Haitao. "Study on Attitude Algorithm of Accelerometers/ Magnetometers Strap-down Inertial Navigation System", Taiyuan: North University of China, Graduate's Thesis, 2007.

[6] Bao Qilian and Wu Zhoujie, "Initial alignment methods of low cost SINS assisted by magnetic compass", in Journal of Chinese Inertial Technology, Vol.16 Num.5,2008, pp.513-518.

[7] Yin Miaomiao, "Research on attitude algorithm of strapdown inertial navigation system", Shanghai: Shanghai Maritime University, Graduate's Thesis, 2007.

[8] China Metorological Administration, Conventional Upper Air Measurement Specification for Operation, Beijing: Metorological press, 2003, pp.2-3. 